Virginia Commonwealth University

VCU Scholars Compass

2008

\title{
Transverse susceptibility study of the effect of varying dipolar interactions on anisotropy peaks in a three-dimensional assembly of soft ferrite nanoparticles
}

Pankaj Poddar

University of South Florida, National Chemical Laboratory

Marienette B. Morales

University of South Florida

Natalie A. Frey

University of South Florida

See next page for additional authors

Follow this and additional works at: http://scholarscompass.vcu.edu/chem_pubs

Part of the Chemistry Commons

Poddar, P., Morales, M. B., \& Frey, N. A., et al. Transverse susceptibility study of the effect of varying dipolar interactions on anisotropy peaks in a three-dimensional assembly of soft ferrite nanoparticles. Journal of Applied Physics, 104, 063901 (2008). Copyright (C) 2008 American Institute of Physics.

\section{Downloaded from}

http://scholarscompass.vcu.edu/chem_pubs/23

This Article is brought to you for free and open access by the Dept. of Chemistry at VCU Scholars Compass. It has been accepted for inclusion in Chemistry Publications by an authorized administrator of VCU Scholars Compass. For more information, please contact libcompass@vcu.edu. 


\section{Authors}

Pankaj Poddar, Marienette B. Morales, Natalie A. Frey, Shannon A. Morrison, Everett E. Carpenter, and Hariharan Srikanth 


\title{
Transverse susceptibility study of the effect of varying dipolar interactions on anisotropy peaks in a three-dimensional assembly of soft ferrite nanoparticles
}

\author{
Pankaj Poddar, ${ }^{1,2, a)}$ Marienette B. Morales, ${ }^{1}$ Natalie A. Frey, ${ }^{1}$ Shannon A. Morrison, ${ }^{3}$ \\ Everett E. Carpenter, and Hariharan Srikanth ${ }^{1, b)}$ \\ ${ }^{1}$ Center for Integrated Functional Materials, Department of Physics, University of South Florida, Tampa, \\ Florida 33620, USA \\ ${ }^{2}$ Materials Chemistry Division, National Chemical Laboratory, Pune 411008, India \\ ${ }^{3}$ Department of Chemistry, Virginia Commonwealth University, Richmond, Virginia 23284, USA
}

(Received 14 April 2008; accepted 10 July 2008; published online 17 September 2008)

\begin{abstract}
Collective magnetization dynamics in nanoparticle assemblies is of current interest as it forms the basis of high density storage media. It is important to understand how interparticle interactions in a three-dimensional (3D) arrangement of superparamagnetic nanoparticles would affect the overall effective magnetic anisotropy of the system. We have studied the influence of varying strengths of dipolar interaction on the static and dynamic magnetic properties of surfactant-coated monodispersed manganese zinc ferrite nanoparticles using reversible transverse susceptibility. We track the evolution of the anisotropy peaks with varying magnetic field, temperature, and interaction strength. The blocking temperature shows an increase from 28 to $32 \mathrm{~K}$ and the coercive field (at 10 K) shows an increase from 144 to 192 Oe as the system changes from the case of weakly interacting to strongly interacting 3D assembly of the particles. (c) 2008 American Institute of Physics. [DOI: $10.1063 / 1.2977592]$
\end{abstract}

\section{INTRODUCTION}

Recent research efforts in magnetically anisotropic materials for media storage at higher densities have demanded the requirement for experimental techniques that can directly probe the magnetic anisotropy and its temperature dependence in a precise manner. There exist several approaches to measure the magnetic anisotropy in fine magnetic particle systems. Examples include the saturation approach, the torsional pendulum, rotational hysteresis, remanent-torque magnetometry, complex permeability spectra, etc. but most of these techniques are indirect and often unwieldy for extracting information about the anisotropy. ${ }^{1-10}$ For example, in the saturation approach, to get the anisotropy constant, one has to do $M-H$ measurements along the hard axis direction and extrapolate the effective anisotropy from the slope change in field-dependent magnetization. However, this is difficult in aggregates of magnetic nanoparticles due to the uniaxial symmetry for spherical particles and distribution in preferred axis directions. ${ }^{6,7}$ Recently, magnetic force microscopy has been shown to yield information about the anisotropy of individual hemispherical nanoparticles grown onto a substrate. ${ }^{11}$ This method, while excellent for determining anisotropy distribution and orientation, is limited to room temperature. Transverse susceptibility (TS), which is a singular point detection technique, has been reasonably successful in assemblies of polycrystalline single-domain particles in probing the anisotropy field in the presence of particle size distribution and texturing (orientation of easy axes). ${ }^{1,2}$

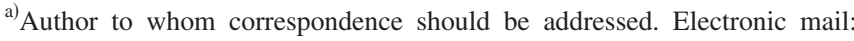
p.poddar@ncl.res.in.

b)Electronic mail: sharihar@cas.usf.edu.
}

The transverse magnetic susceptibility for a singledomain uniaxial particle was first calculated by Aharoni et $a l .{ }^{12}$ within the framework of the Stoner-Wohlfarth model in 1957. For a unipolar field scan from positive to negative saturation, the theoretical curve for TS exhibits three singularities. Two of these are located precisely at the effective anisotropy field $\left(H_{K(\text { eff })}=H_{K(\text { cryst })}+H_{K(\text { (shape })}\right)$ and the third peak is located at the switching field. ${ }^{3}$ It is worth noting that for a system with a collection of randomly oriented particles, the sharpness of the singular peaks are greatly reduced due to the distribution in $H_{K}$, but nevertheless, it is still very much observable in experiments. ${ }^{13-15}$

In TS experimental configuration, a small amplitude ac or rf field is typically introduced perpendicular to the dc field. ${ }^{12,13}$ The effect of the transverse field $(\Delta H)$ is equivalent to rotating the static field $H$ in the same direction by an angle of $\Delta H / H$. TS is related to the second derivative of free energy $(F)$ and is essentially a measure of the "curvature" of $F$ or of the stiffness of $M$ (magnetization). In fact, it has been argued that TS is also described as the zero frequency limit of the ferromagnetic resonance. ${ }^{16}$

An assembly of noninteracting Stoner-Wohlfarth nanoparticles can be considered as a system where their easy axes are randomly oriented. For such a system, we expect that the singularities located at $\pm H_{K}$ (which we refer to as the anisotropy peaks) will depend only on the contributions of the nanoparticles oriented with their easy axis nearly perpendicular to the dc magnetic field $\left(H_{\mathrm{dc}}\right)$. Ideally the TS peaks are located at $H_{K}$, however small deviations are seen due to shapes of nanoparticles, local inhomogeneities of the demagnetizing field, etc. Unlike the shape, the position of the anisotropy peaks must be independent of the distribution in size 
and orientation of the crystallites even when the average crystallite dimensions are well above the single-domain particle size. ${ }^{17}$

Though a wealth of literature are available on measurements and modeling of magnetic anisotropy using the TS technique, only very few studies present systematic measurements of the effect of interparticle dipolar/exchange interactions on the effective anisotropy peaks. Recently, Presa et $a l .{ }^{18}$ used a mean field model to substitute the applied magnetic field with an effective magnetic field due to the interparticle interactions and showed that for an in-plane isotropic assembly of randomly oriented (110) uniaxial particles, the TS peak shifts toward lower magnetic field values with increase in the interaction between the particles. Chang and Yang ${ }^{19}$ reported a similar effect on the randomly oriented uniaxial particles in another theoretical study. The same work showed that the interaction effects could lead to the anisotropy peaks gradually merging with the coercivity (or switching) peak at $H_{C}$ with increasing interparticle interactions.

Most of the experimental studies in the past were affected by complications associated with poor control over size and shape of the particles, magnetic texturing, strong exchange coupling, bulk interaction effects due to agglomerations, etc. ${ }^{20-22}$ In a previous study on hard magnetic materials, it was shown that TS peak position and shape do not depend at all on the interaction between the grains. ${ }^{1}$ In another study on tapes of $\mathrm{CrO}_{2}$ particles inside an epoxy, it was found that the peak position $H_{K}$ decreases with increase in the packing fraction (i.e., with increase in the interaction strength). However this study was affected by inherent texturing due to the sample processing in form of a tape. ${ }^{21} \mathrm{Tex}-$ turing also has a significant effect on the TS peak position. Therefore, this study could not isolate the two effects. The agglomeration and clustering effects may also have affected this study which was on rod-shaped $\mathrm{CrO}_{2}$ particles with lengths of $300 \mathrm{~nm}$ and an aspect ratio of 10:1. With the advancements in wet-chemical synthesis techniques in recent years, it has been possible to achieve desired control over the particle size, shape, and their assembly in different concentrations within a host matrix.

To study the effect of varying strength of dipolar interactions on TS peaks in a reasonably controlled fashion, we used high quality, chemically synthesized, monodispersed manganese zinc ferrite (MZFO) nanoparticles as a model system and varied their concentration in paraffin wax matrix, which is a nonmagnetic host. The variation in coercivity and blocking characteristics as observed through dc susceptibility experiments were measured and compared with TS experiments. We were thus able to make a systematic study of the influence of interparticle interactions directly on the anisotropy peaks.

\section{EXPERIMENTAL RESULTS AND DISCUSSIONS}

Monodispsered MZFO $\left(\mathrm{Mn}_{0.68} \mathrm{Zn}_{0.25} \mathrm{Fe}_{0.27} \mathrm{O}_{3}\right)$ nanoparticles were synthesized using the reverse micelle method using bis-(2-ethylhexl) sodium sulfosuccinate (AOT) as surfactant medium. These particles were extensively characterized

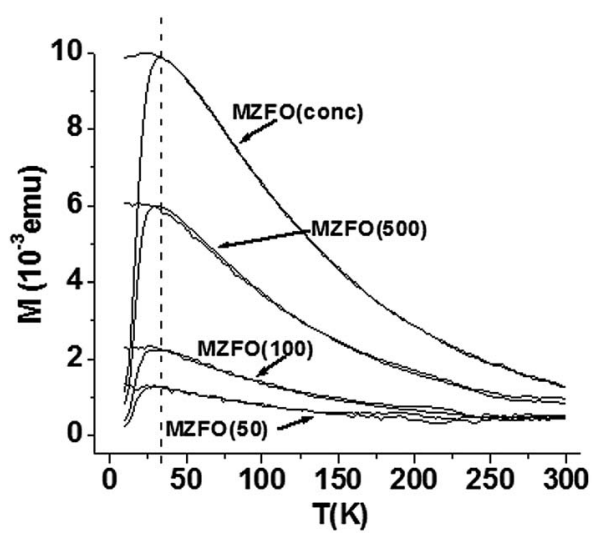

FIG. 1. Zero field cooled (ZFC) and field cooled (FC) magnetization at 100 Oe for various samples. Straight line is guide to the eye. The inset shows the data near the blocking temperature.

for their size and crystalline phase using TEM, extended $\mathrm{x}$-ray-absorption fine structure, and x-ray diffraction. They were found to be single crystalline with an average size of 15 $\mathrm{nm}$ and a standard deviation of less than $10 \%$. Further details are presented in Ref. 14. For magnetic property measurements, the MZFO particles coated with excess surfactant and suspended in $n$ propanol were dispersed in varying concentrations in pure paraffin wax to alter the average interaction strength in a reasonably controlled manner. The samples were sonicated in molten form to allow uniform dispersion of the particles and evaporation of the solvent. Finally the wax was solidified while sonicating in order to avoid any unwanted texturing and to ensure random orientation of the easy axes. Agglomeration of the particles was eliminated by the presence of excess surfactant (AOT) as well as wax medium surrounding individual particles. Using this process, we prepared three samples labeled MZFO(50), MZFO(100), and MZFO(500) by dispersing 50, 100, and $500 \mu \mathrm{l}$ of solvent containing the MZFO nanoparticles, respectively, in same amount of wax. The solidified chunks of samples were packed inside gelatin capsules and used for both static and dynamic magnetic measurements. A fourth sample, MZFO(conc) was prepared by air drying the solvent (without any wax) inside the gel cap in ambient conditions leaving a thick deposit of nanoparticles at the bottom of the gel cap so as to get the maximum concentration of particles representing the case of highest magnitude of interparticle interactions relative to the other samples.

Dc magnetization measurements were performed using a physical property measurement system from Quantum Design equipped with a 7 Tesla superconducting magnet. Zero field cooled (ZFC) and field cooled (FC) measurements were done at 100 Oe magnetic fields. In Fig. 1, we have compared between ZFC-FC data for the samples $\mathrm{MZFO}(50)$, MZFO(100), MZFO(500), and MZFO(conc). It can be noticed that all the samples show the blocking peak characteristic of the high temperature superparamagnetic nature of the particles. The sharpness of the peak reflects the quality of monodispersity of the particles. A broad transition would indicate the presence of considerable size distribution. The sharpness observed in the data indicates that wax does not facilitate agglomeration of the particles in contrast to media 
TABLE I. The blocking temperature and low temperature coercivity of the MZFO samples.

\begin{tabular}{lccc}
\hline \hline \multicolumn{1}{c}{ Sample } & Decription & $T_{B}$ & $H_{C}(\mathrm{Oe})$ at $10 \mathrm{~K}$ \\
& & $(\mathrm{~K})$ & \\
\hline MZFO (50) & $50 \mu$ l of nanoparticles dispersed in wax & 28 & 144 \\
MZFO (100) & $100 \mu$ l of nanoparticles dispersed in wax & 29 & 163 \\
MZFO (500) & $500 \mu$ l of nanoparticles dispersed in was & 30 & 178 \\
MZFO (conc) & Nanoparticle in solution dried inside & 32 & 192 \\
& a gelcap to form a thick layer & & \\
\hline \hline
\end{tabular}

such as polymers used in several studies. It has been shown that the normal tendency of particles in polymer matrices is to agglomerate because of the strong steric forces and their effect on the magnetic properties could be easily observed. ${ }^{14}$ In a following study, we have shown how this agglomeration of the nanoparticles in polymers can be overcome using careful choice of solvent and surfactant mixtures but these complications are not present in paraffin wax used in this study so we do not need special procedures. ${ }^{23}$

The blocking transition region, highlighted in the inset of Fig. 1, shows that the blocking temperature peak increases with increasing packing fraction from $28 \mathrm{~K}$ for $\mathrm{MZFO}(50)$ to $32 \mathrm{~K}$ for MZFO(conc). In Table I, we have summarized the measured magnetic parameters. It should be pointed out that in this system, as the particles have surfactant coating and being dispersed in wax, the interactions are mostly dipolar in nature as opposed to direct exchange interactions. The effect of the particle concentration on the blocking temperature reflects the role of interparticle interaction affecting the relaxation behavior and is consistent with other studies. ${ }^{24}$

In Fig. 2, we have compared the $M-H$ hysteresis loops for all the samples at $300 \mathrm{~K}$ (top panel) and at $10 \mathrm{~K}$ (bottom panel), respectively. The $M-H$ curves at $300 \mathrm{~K}$ show revers-
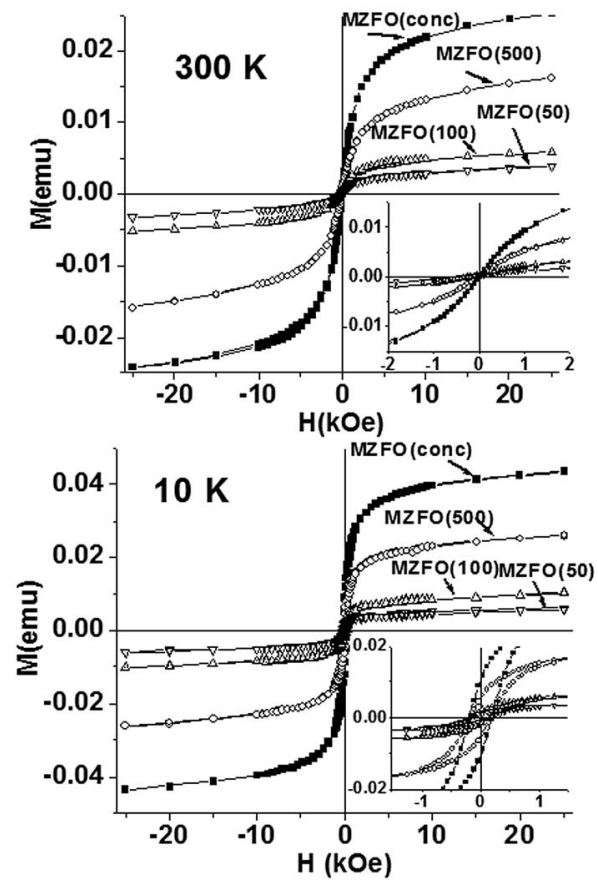

FIG. 2. Top panel: Magnetization vs field curves at $300 \mathrm{~K}$. The inset shows the zoom view of the curves. Bottom panel: Magnetization vs field curves at $10 \mathrm{~K}$. The inset shows the zoom view of the curves. ible behavior indicating the superparamagnetic state of the particles. At $10 \mathrm{~K}$, a systematic increase in the coercivity with increasing packing fraction (from 144 to 192 Oe) can be seen which clearly shows an effect of interparticle interaction strength. This increase in coercivity is consistent with increase in the blocking temperature. The concentration dependence (packing fraction of particles inside a matrix) of the net field, affecting the magnetization reversal within a typical particle is given by the following relation: ${ }^{25}$

$$
H_{\text {tot }}=H_{\text {appl }}-\left(v N_{d}^{\text {sample }}+N_{d}^{\text {particle }}+\gamma\right) M_{P},
$$

where $H_{\text {appl }}$ is the applied field, second term is the sample demagnetizing field, related to the sample magnetization and the sample shape through a demagnetizing factor $N_{d}^{\text {sample }}(v$ is the packing fraction), third term is the particle demagnetizing field, related to the typical particle magnetization $M_{P}$ and particle shape through a demagnetization factor $N_{d}^{\text {particle }}$, and the last term is represented by the interparticle interaction field, depending on the magnetization and distance of nearby particles characterized by an interaction field parameter $\gamma$. Here the change in $\left(N_{d}^{\text {particle }}+\gamma\right) M_{P}$ with particle concentration (or packing fraction $v$ ) is equivalent to the change in local field affecting the magnetization reversal of a particle. ${ }^{25}$ The value of $N_{d}^{\text {particle }}$ is very small and can be neglected because of the slightly ellipsoidal shape of the particles. ${ }^{17}$

To further probe the effect of interactions on the magnetic response, we conducted TS experiments using a resonant $\mathrm{rf}$ technique based on a tunnel-diode oscillator. ${ }^{26}$ In this method, the gel cap containing the particles is placed inside the inductance coil of an LC tank circuit which is selfresonant at $12 \mathrm{MHz}$ (Fig. 3 top panel). The rf current flowing through the windings of the inductance coil, which is placed inside the PPMS, provides the rf perturbing field which is transverse to the dc field. In Fig. 3 (bottom panel), we have shown the schematic representation of the magnetization vector subject to the dc and rf fields. ${ }^{13}$ As described by the Aharoni theory and many of TS experiments in the past, it should be emphasized that only in the transverse geometry one could resolve the well-defined anisotropy peaks. On the other hand, doing the experiment with rf and dc fields parallel to each other would just give the standard field-dependent parallel susceptibility (i.e., effectively the first derivative of the $M-H$ hysteresis loop). ${ }^{17}$ The TS measurements were performed under ZFC conditions with the data taken while warming up the sample.

In Fig. 4, we have shown representative TS curves for samples MZFO(100) (bottom panel) and MZFO(conc) (top panel). In these panels, the family of curves traces the evo- 

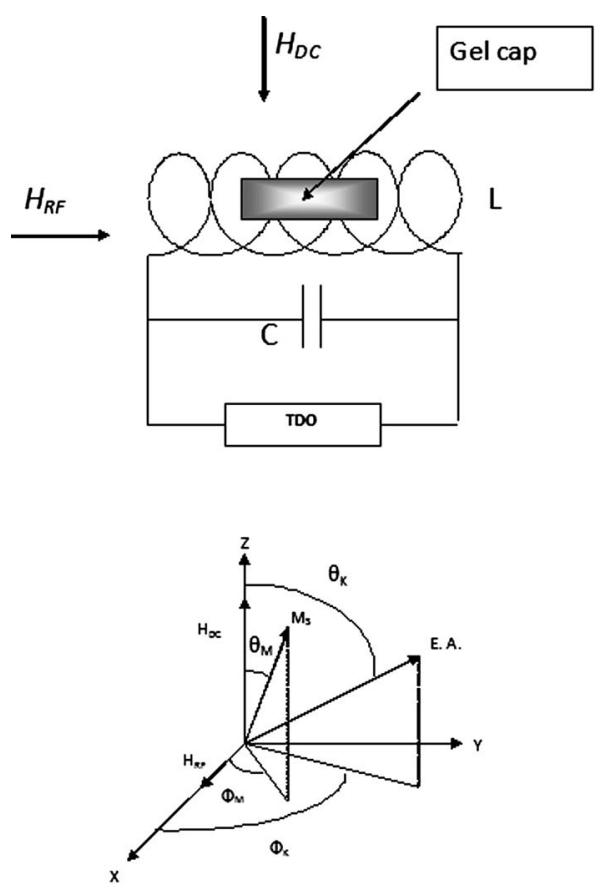

FIG. 3. (Top) Schematic of the tunnel-diode oscillator (TDO) based transverse susceptibility (TS) measurement geometry. (Bottom) Schematic representation showing magnetization, easy axis, rf, and dc field directions for the TS configuration.

lution of the system as the temperature is increased. As can be noticed in Fig. 4, when the temperature is lowered, the single peak in the TS at zero field splits into two peaks, which become more prominent with decreasing temperature and shift to higher field values. It is also clear from both the panels in the Fig. 4 that the overall shape of the TS curves including the peak positions is remarkably different for the two extreme cases of MZFO(conc) and MZFO(100). To follow these differences in features in the TS closely, we have compared in Fig. 5 the unipolar scans (from positive saturation to negative saturation) for different samples at four fixed temperatures. In the upper panel of Fig. 6, we have plotted the peak position versus temperature curves for all the four samples in both first and second quadrant (of the unipolar scan). The peak positions in both the quadrants are almost symmetric to each other and strongly dependent on temperature. Again from the curves in the Fig. 5, it can be observed that at $15 \mathrm{~K}$, the peaks corresponding to $\mathrm{MZFO}$ (conc) are situated at larger field values in comparison with the other samples $\quad[\mathrm{MZFO}($ conc $)>\operatorname{MZFO}(500)>\mathrm{MZFO}(100)$ $>\mathrm{MZFO}(50)]$. This shows that the values of effective anisotropy peak positions, as obtained from TS curves, are strongly and systematically affected by interparticle dipolar interactions and increase with the strength of the interparticle interactions. Our experimental results are thus in contrast to the conclusions of the earlier theoretical studies that predicted a decrease in the anisotropy fields with enhanced interactions. ${ }^{18,19,27,28}$

Another important feature of TS curves is the height and shape of the TS peaks located at $\pm H_{K}$. As observed from Fig. 5, in the TS experiments, when going from positive saturation to the negative saturation field values, the peak

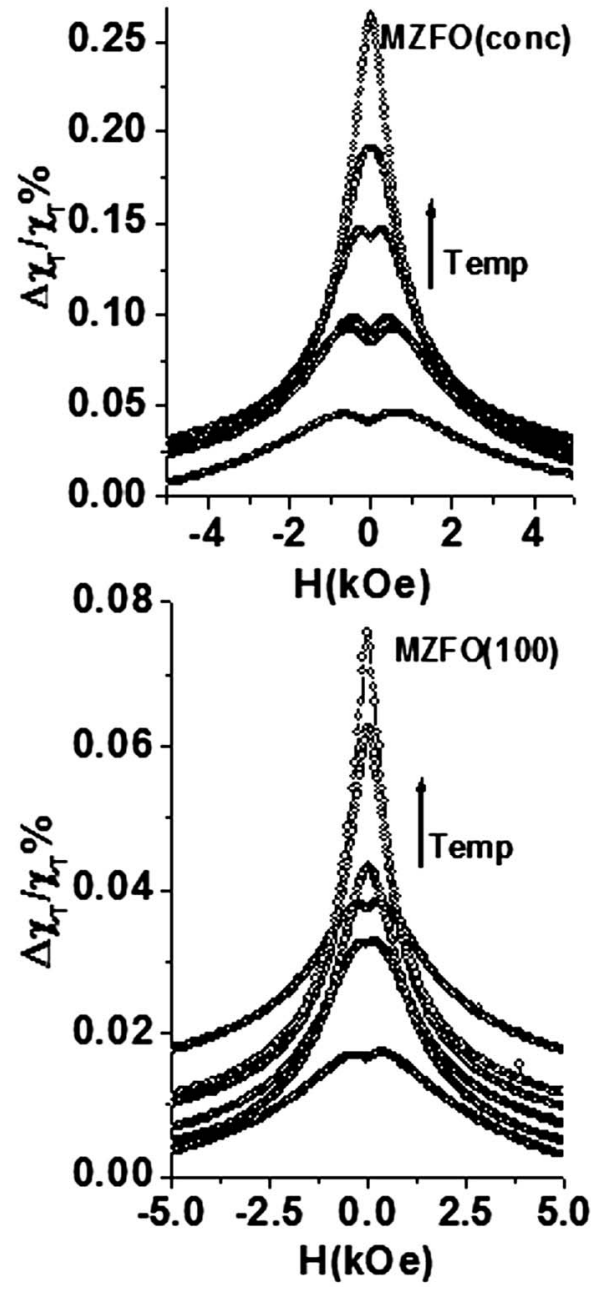

FIG. 4. Representative TS curves for (a) MZFO(conc) and (b) MZFO(100) samples taken at $10,20,25,35,45$, and $60 \mathrm{~K}$. The arrow indicates curves measured at increasing temperatures.

height in the first quadrant is always larger than the peak height in the second quadrant. This is a fact that we have consistently observed in our previous studies as well. ${ }^{13-16}$ However, it was unclear what causes the difference in the height of the peaks and the systematic studies here on samples of varying interaction strengths allows us to explore the origin of the peak height symmetry.

To better express the issue of peak height quantitatively, in the lower panel of Fig. 6, we have plotted the peak height difference

$\left[(\text { Peak height })_{\text {first quadrant }}\right.$ $\left.-(\text { Peak height })_{\text {second quadrant }} /(\text { Peak height })_{\text {first quadrant }} \%\right]$ as a function of sample temperature. It is remarkable to see (from Figs. 5 and 6) that for the most concentrated sample, the peak height difference is highly reduced in comparison to the weakly interacting particle system. Our results indicate that the difference in peak heights (located at $\pm H_{K}$ ), or the asymmetry, is also extremely sensitive to a change in the dipolar interaction strength and increases with decreasing interaction. In a recent theoretical investigation by Matarranz et $a l .{ }^{30}$ it was claimed that for a textured assembly of uniaxial single-domain interacting particles, the dispersion in the anisotropy field (particle size, shape) causes the peak height asymmetry and broadening. They calculated that that for a 

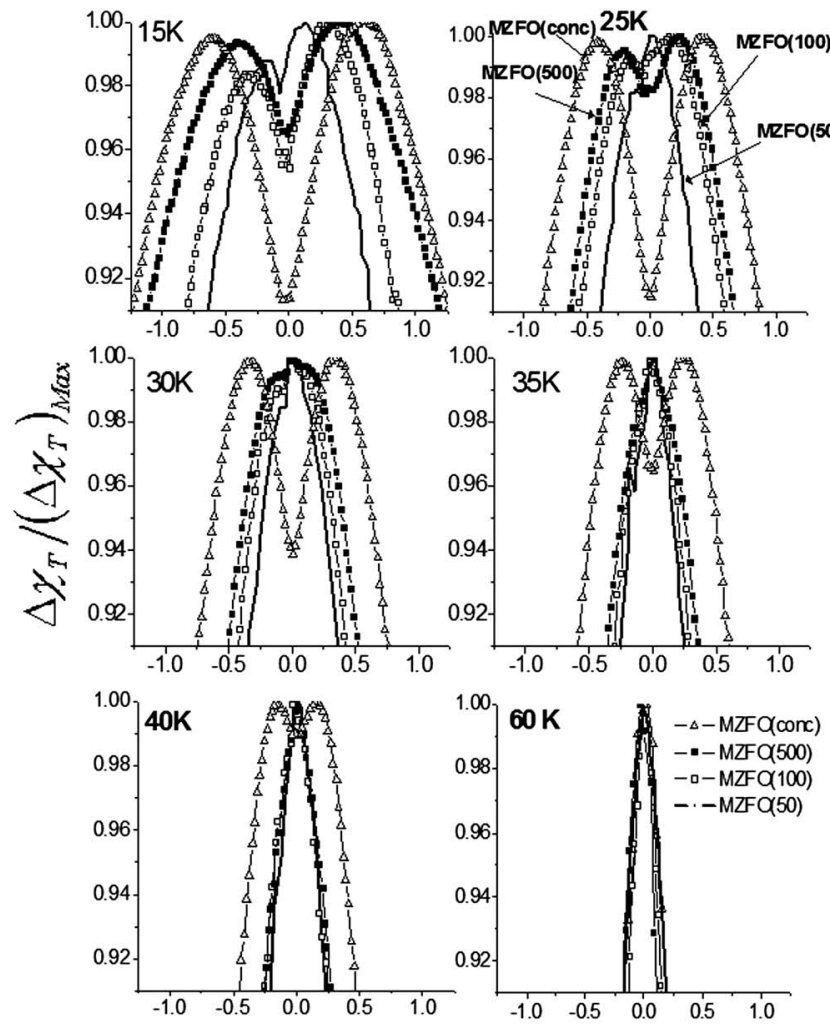

\section{$H(k O e)$}

FIG. 5. Comparison of TS curves at various concentrations and temperatures.

monodispersed assembly of particles, the TS peaks would be very sharp and similar in height. However, our experimental study is in sharp contrast to this and the trend is the opposite. Note that in our case we have taken particles from the same synthesis batch and all other experimental conditions remain the same for all the samples except for the concentration of the particles in wax that determines the interparticle interactions. Therefore, we infer that the anisotropy field dispersion remains constant for all the samples. We also conclude that the change in the peak height difference is not due to the distribution of the anisotropy field alone as indicated by previous theoretical studies but the interparticle interaction also plays a major role. Overall, we demonstrate that TS is sensitive enough to pick up small changes in the interaction strength.

We now present arguments that will help qualitatively understand the evolution of the peak height asymmetry from large to small as the nanoparticle system goes from weakly to strongly interacting cases. Recall that in the unipolar TS scans from positive to negative saturation for $\mathrm{MZFO}(50)$ or MZFO(100), the higher peak occurs on the positive side and the lower peak on the negative side. This may be understood from the perspective of competition between Zeeman energy (which dominates at high field and causes forcible alignment of moments in the field direction) to the anisotropy energy (which dominates near zero field). In the latter case, the situation is very different for noninteracting particles where the distribution in anisotropy fields is truly random whereas for
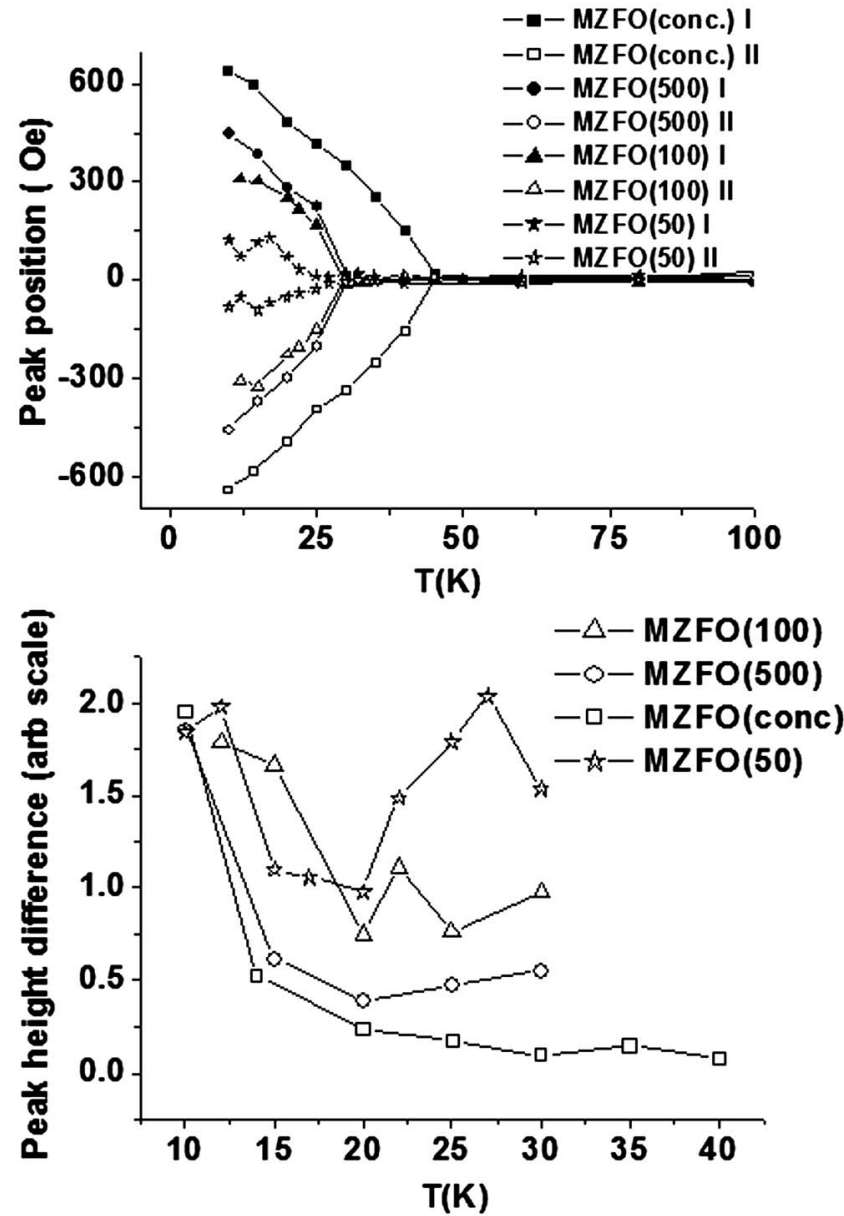

FIG. 6. (a) Anisotropy peak height difference (arbitrary scale) vs temperature at various concentrations. (b) Peak position $\left(H_{K}\right)$ vs temperature in both the quadrants for various concentrations. The field positions of the anisotropy peaks in both quadrants (presented as I for the first quadrant and II for the second quadrant) as a function of temperature.

interacting particles, considerable short range order is present resulting in smaller distribution in effective anisotropy fields of the clusters. The distribution in anisotropy fields resulting from a considerable size distribution in particles (i.e., a polydispersed sample) will also manifest as an asymmetry in peak width, with the second peak appearing broadened as well as smaller in height. This is not so evident in the highly monodispersed sample we used for this study, but is a feature often seen in polydispersed nanoparticle systems. ${ }^{13-16}$ Thus, in general, when the field is reversed in polarity, the height and width of the second peak on the negative side are different for the two cases (i.e., small and broad for the case of noninteracting particles versus larger and sharper for the case of interacting particles) and depends on the different energy landscapes seen by the noninteracting and interacting particles at zero field (demagnetized state). This explains the TS peaks being symmetric in height for samples with strong interactions and displaying a large asymmetry for noninteracting/weakly interacting particles. Our explanation is also very consistent with TS data on ferromagnetic thin films which always display sharp peaks symmetric in height. ${ }^{29,30}$ In the case of thin films, one can effectively con- 
sider them as strongly interacting systems with no distribution in anisotropy fields as the easy and hard axes are well defined.

In summary, we have investigated the influence of increasing dipolar interactions on the blocking temperature, coercivity, and the magnetic anisotropy in monodispersed MZFO nanoparticles. TS has been used to directly probe the effect of inter-particle interactions on the anisotropy and it is observed that the anisotropy fields increase with increasing interactions. The asymmetry in TS peaks is also associated with the strength of interactions with weakly interacting particles exhibiting strong asymmetry in the TS anisotropy peaks whereas they are symmetric for strongly interacting particles.

\section{ACKNOWLEDGMENTS}

The Center for Integrated Functional Materials (CIFM) at the University of South Florida is supported through Grant No. USAMRMC-07355004. H.S. also acknowledges support from U.S. DOE Basic Energy Sciences through Grant No. DE-FG02-07ER46438. P.P. thanks financial support by the Department of Science and Technology, India through Grant No. SR/S5/NM-104/2006 under the "Nano Mission" program.

${ }^{1}$ C. Asti and S. Rinaldi, J. Appl. Phys. 45, 3600 (1974).

${ }^{2}$ R. O'Handley, Modern Magnetic Materials: Principles and Applications (Wiley, New York, 1999).

${ }^{3}$ V. Franco and A. Conde, J. Magn. Magn. Mater. 278, 28 (2004).

${ }^{4}$ G. Asti and S. Rinaldi, Phys. Rev. Lett. 28, 1584 (1972).

${ }^{5}$ Y. Endo, O. kltakami, S. Okamoto, and Y. Shimada, Appl. Phys. Lett. 77, 1689 (2000).

${ }^{6}$ F. Zhou and K. Y. He, J. Appl. Phys. 79, 8850 (1996).

${ }^{7}$ K.-Y. Ho, X.-Y. Xiong, J. Zhi, and L.-Z. Cheng, J. Appl. Phys. 74, 6788
(1993).

${ }^{8}$ G. Turilli, J. Magn. Magn. Mater. 130, 377 (1994).

${ }^{9}$ C. Papusoi, Jr. and T. Suzuki, J. Magn. Magn. Mater. 240, 568 (2002).

${ }^{10}$ G. Bottoni, D. Candolfo, and A. Cecchetti, J. Appl. Phys. 81, 3794 (1997).

${ }^{11}$ H. Krishna, C. Miller, L. Longstreth-Spoor, Z. Nussinov, A. K. Gangopadhyay, and R. Kalyanaraman, J. Appl. Phys. 103, 073902 (2008).

${ }^{12}$ A. Aharoni, E. H. Frei, S. Shtrikman, and D. Treves, Bull. Res. Counc. Isr. 6A, 215 (1957).

${ }^{13}$ P. Poddar, J. L. Wilson, H. Srikanth, D. F. Farrell, and S. A. Majetich, Phys. Rev. B 68, 214409 (2003).

${ }^{14}$ P. Poddar, J. L. Wilson, H. Srikanth, S. A. Morrison, and E. E. Carpenter, Nanotechnology 15, S570 (2004).

${ }^{15}$ P. Poddar, Y. Sahoo, H. Srikanth, and P. N. Prasad, Appl. Phys. Lett. 87, 062506 (2005).

${ }^{16}$ L. Spinu, I. Dumitru, A. Stancu, and D. Cimpoesu, J. Magn. Magn. Mater. 296, 1 (2006).

${ }^{17}$ L. Spinu, H. Pham, C. Radu, J. C. Denardin, I. Dumitru, M. Knobel, L. S. Dornelesc, L. F. Schelp, and A. Stancu, Appl. Phys. Lett. 86, 012506 (2005).

${ }^{18}$ B. Presa, R. Matarranz, J. F. Calleja, and M. C. Contreras, J. Appl. Phys. 99, 08Q511 (2006).

${ }^{19}$ C.-R. Chang and J.-S. Yang, Appl. Phys. Lett. 65, 498 (1994).

${ }^{20}$ P. M. Sollis, P. R. Bissell, and R. W. Chantrell, J. Magn. Magn. Mater. 155, 123 (1996)

${ }^{21}$ P. M. Sollis, P. R. Bissell, and Y. Matsutake, IEEE Trans. Magn. 33, 3046 (1997).

${ }^{22}$ G. Zimmermann, J. Appl. Phys. 77, 2097 (1995).

${ }^{23}$ J. Gass, P. Poddar, J. Almand, S. Srinath, and H. Srikanth, Adv. Funct. Mater. 16, 71 (2006).

${ }^{24}$ T. Jonsson, J. Mattsson, C. Djurberg, F. A. Khan, P. Nordblad, and P. Svedlindh, Phys. Rev. Lett. 75, 4138 (1995).

${ }^{25}$ G. J. Tomka, P. R. Bissel, R. W. Chantrell, and K. O'Grady, IEEE Trans. Magn. 29, 2869 (1993).

${ }^{26}$ H. Srikanth, J. Wiggins, and H. Rees, Rev. Sci. Instrum. 70, 3097 (1999). ${ }^{27}$ Ching-Ray Chang and J.-S. Yang, IEEE Trans. Magn. 30, 4095 (1994).

${ }^{28}$ R. Matarranz, M. C. Contreras, G. Pan, B. Presa, J. A. Corrales, and J. F. Calleja, J. Appl. Phys. 99, 08Q504 (2006).

${ }^{29}$ L. Spinu, H. Srikanth, A. Gupta, X. W. Li, and G. Xiao, Phys. Rev. B 62, 8931 (2000).

${ }^{30}$ N. A. Frey, S. Srinath, H. Srikanth, M. Varela, S. Pennycook, G. Miao, and A. Gupta, Phys. Rev. B 74, 024420 (2006). 